Ocular Oncology

and Pathology
Ocul Oncol Pathol 2018;4:165-169

DOI: $10.1159 / 000481533$
Received: May 29, 2017

Accepted after revision: September 13, 2017

Published online: November 4, 2017

\title{
Congenital Orbital Rhabdomyosarcoma
}

\author{
Masoomeh Eghtedari ${ }^{a} \quad$ Amir Reza Farsiani ${ }^{a} \quad$ Mohammad Reza Bordbar ${ }^{b}$ \\ a Poostchi Ophthalmology Research Center, Department of Ophthalmology, and ${ }^{\mathrm{b}}$ Hematology Research Center, \\ School of Medicine, Shiraz University of Medical Sciences, Shiraz, Iran
}

\section{Established Facts}

- Orbital rhabdomyosarcoma is rarely presented at birth. This tumor should be considered when facing unilateral proptosis in a newborn.

- Detection of intracranial extension or systemic involvement requires a comprehensive workup including imaging.

\section{Novel Insights}

- Despite treatment, prognosis for life was poor, mainly due to early recurrence.

- This fact should be considered in decision making for orbital reconstruction and any cosmetic procedure.

\section{Keywords}

Rhabdomyosarcoma · Orbit · Congenital tumor .

Exenteration

\section{Abstract}

Rhabdomyosarcoma (RMS) is the most common malignant tumor of the orbit in children, but it is rarely present at birth. We report a large congenital orbital RMS with intracranial extension in a newborn baby. A newborn baby girl was referred to our hospital due to severe right-eye exophthalmia. Imaging studies showed an orbital mass with intracranial extension. Treatment was started with a course of neoadjuvant chemotherapy followed by right orbital exenteration and intracranial resection of the tumor. Histologic examination of the mass showed undifferentiated malignant small-cell tumor. Immunohistochemical study proved it to be RMS. The patient was categorized as intermediate-risk RMS and chemotherapy was continued accordingly with VAC regimen. In spite of treatment, the infant developed intracranial recurrence at the age of 6 months and died 1 month later. Congenital orbital RMS has a poor prognosis. Reconstruction surgery should be deferred due to high rate of recurrence and low chance of survival. A multidisciplinary approach might increase the survival of these patients.

(C) 2017 S. Karger AG, Base

\section{KARGER}

(c) 2017 S. Karger AG, Basel

E-Mail karger@karger.com

www.karger.com/oop
Amir Reza Farsiani, MD

Poostchi Ophthalmology Research Center

Zand Street

Shiraz 7134997446 (Iran)

E-Mail amirrezafarsiani@gmail.com 


\section{Introduction}

Orbital rhabdomyosarcoma (RMS) arises from pluripotent mesenchymal cells and is the most common orbital malignancy in children. It accounts for approximately $4 \%$ of orbital masses in this age group. The mean age of diagnosis is $6-8$ years; however, it is very rare at birth $[1,2]$.

Although orbital RMS can be either intraconal, extraconal, or both, invasion to the globe or intracranial exten- sion is very rare. Histologically, most orbital RMS in children are generally embryonal or alveolar [2]. In recent decade, timely diagnosis and a more effective chemotherapy regimen have led to a surge in survival.

\section{Case Report}

A newborn baby girl was referred to the emergency room of our hospital after being delivered through an uneventful vaginal delivery with right-side exophthalmic eye. Both parents were
Fig. 1. a, b Significant exophthalmia on the right side. Subconjunctival hemorrhage and lid ecchymosis are visible. Cornea seems to be clear at birth. Postoperative follow-ups at the age of 2 (c) and 4 (d) months.

Fig. 2. a Axial orbital CT scan without contrast showed large retrobulbar isodense mass. b Axial T2-weighted MR image of orbit revealed large retrobulbar heterogeneous mass. Both images showed tumoral extension to the middle cranial fossa, nasal cavity, and sphenopalatine fossa.
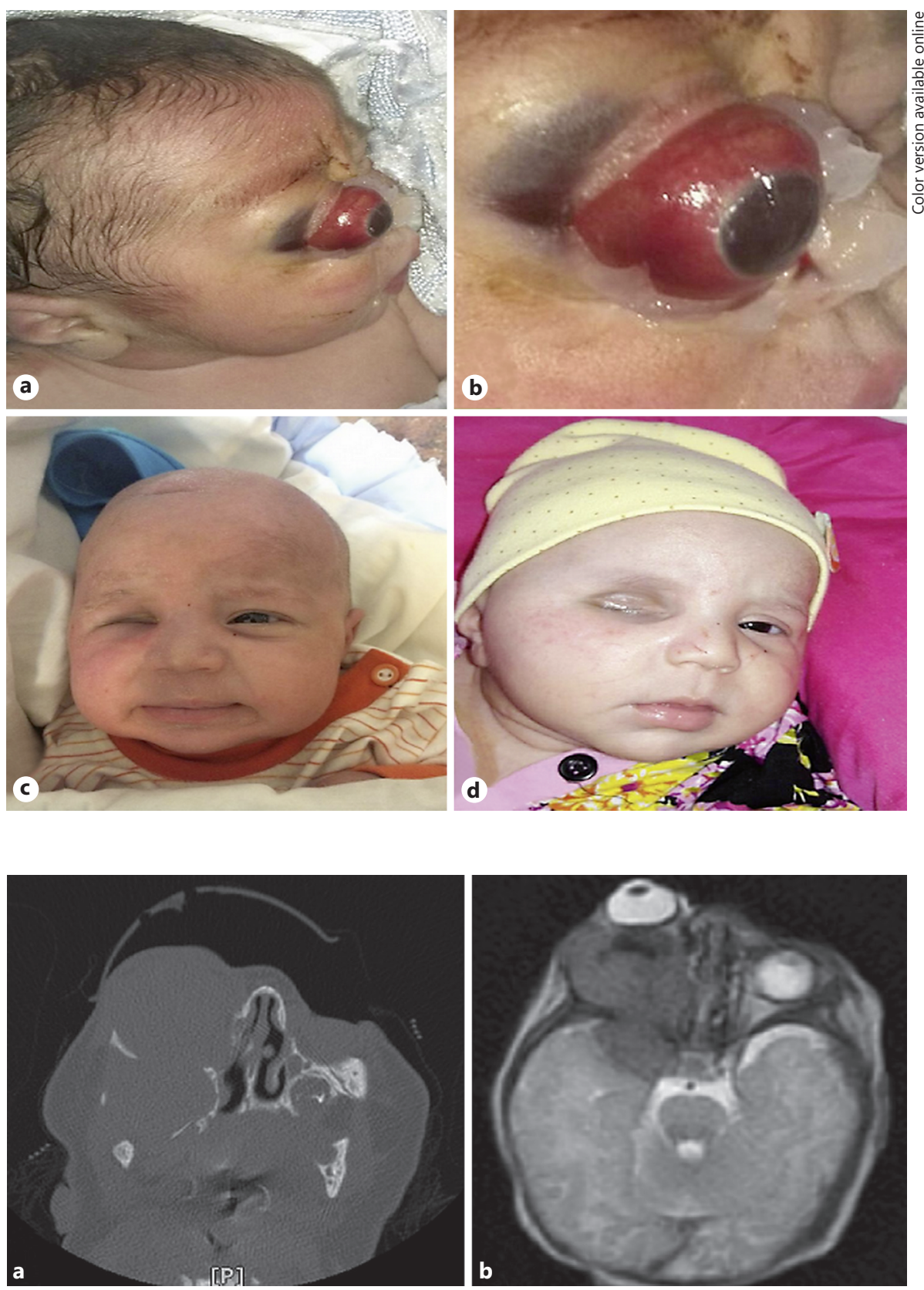
healthy, and the mother had experienced a normal course of pregnancy. Prenatal ultrasound at the gestational age of 20 weeks was reported to be normal; however, no recorded document was available for review.

Upon clinical examination, significant proptosis of the right eye along with lid swelling, subconjunctival hemorrhage, chemosis, and periorbital skin ecchymosis were observed (Fig. 1a, b). Her pupils had sluggish response to light, but no relative afferent pupillary defect was detected. Fundus examination showed mild disk margin blurring with normal foveal reflex in the affected eye. Upon examination, her left eye was normal and no lymphadenopathy or any systemic abnormality was detected.

Systemic workup including complete blood count, urinalysis, serum electrolytes, creatinine level, liver function tests, bone marrow aspiration, lumbar puncture, abdominal ultrasound, and chest X-ray was unremarkable. However, orbital imaging and brain examination including CT scan and MRI showed a large retrobulbar inhomogeneous and irregularly enhanced mass with an extension into the right middle cranial fossa, nasal cavity, and sphenopalatine fossa (Fig. 2) suggestive of a malignant process.

Neoadjuvant treatment consisting of vincristine, cyclophosphamide, and actinomycin D was initiated in order to reduce the tumoral tissue before surgery, and excision was performed a week later. The goal was to debulk the tumor as much as possible, while preserving normal-appearing tissues. Right frontotemporal craniotomy was done and the tumor was exposed through a subfrontal approach. Tissue sample was sent for rapid diagnosis via frozen section method. Prepared sections revealed highly malignant small-cell tumor with extensive hemorrhage areas and necrosis. Orbital exploration was in favor of blending tumoral tissue around the globe. Extraocular muscles were adherent to the tumor and optic nerve was trapped in the abnormal tumoral tissue. In spite of debulking, any effort to put the globe back into its normal position failed; hence, modified exenteration with preserva-
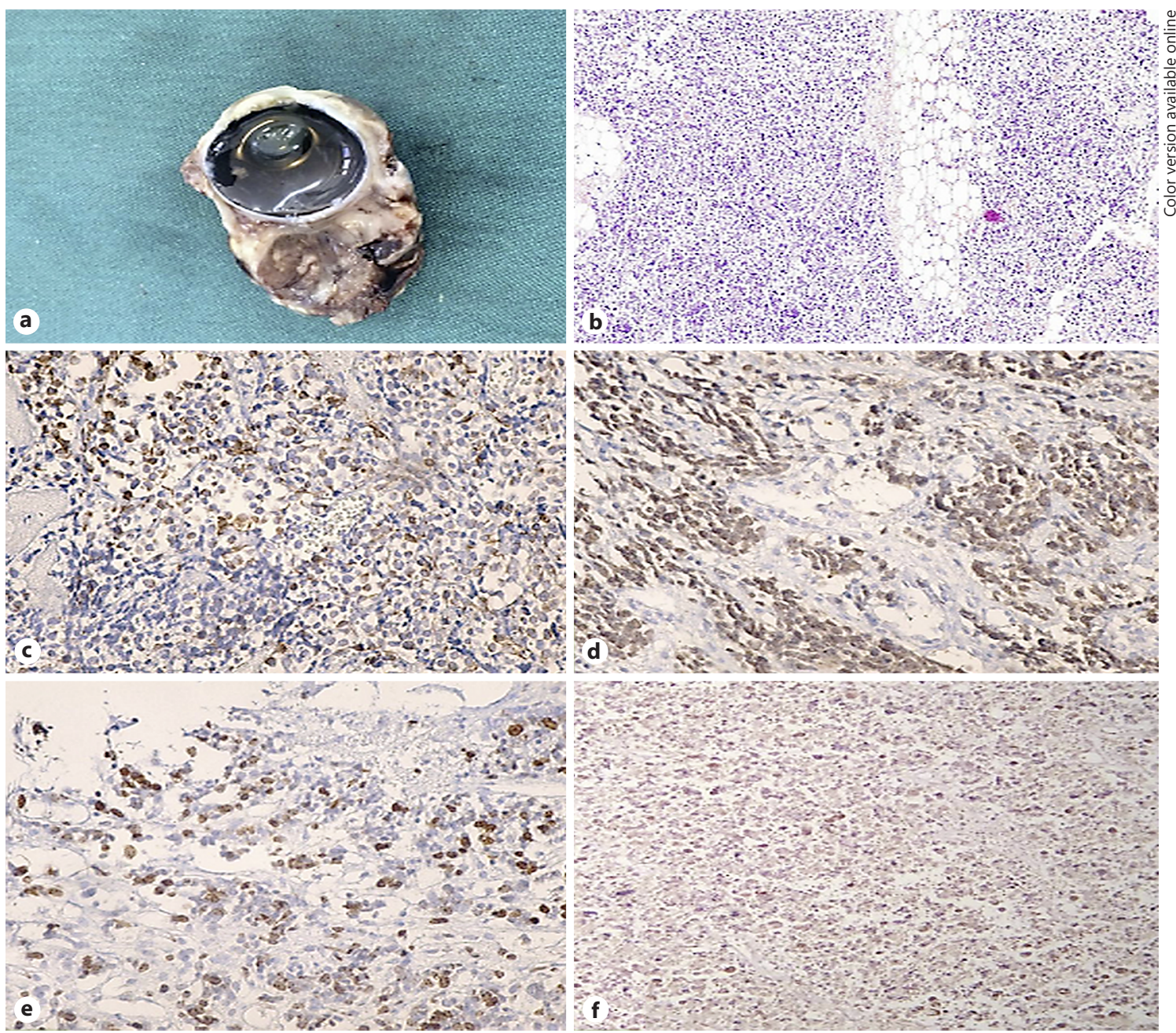

Fig. 3. a Gross appearance of tumor: Tumoral tissue is around the globe. b Malignant small tumoral cells with areas of hemorrhages and necrosis $(\mathrm{H} \& \mathrm{E}, \times 40)$. Positive immunohistochemistry staining for desmin $(\mathbf{c})$, MyoD1 (d), and Ki67 (e); mutation in P53 was almost negative (f) $(\times 400)$. 
tion of lids was the only procedure we could perform. Written informed consent was obtained from her parents. Finally, residual tumoral tissue in the temporal fossa was cleared out and she was transferred to the intensive care unit, where she experienced a smooth recovery.

Gross appearance of the orbital content is shown in Figure 3a. Microscopic examination of the tissue showed malignant small tumoral cells lacking differentiation along with large areas of hemorrhages and necrosis (Fig. 3b). Immunohistochemistry study is shown in Figure $3 \mathrm{c}-\mathrm{f}$.

Based on the Intergroup Rhabdomyosarcoma Study, this patient was classified as stage 3 , group III, intermediate risk group. Accordingly, VAC/VTC regimen (vincristine, actinomycin D, and cyclophosphamide, alternating with vincristine, topotecan, and cyclophosphamide) was indicated and the Children's Oncology Group (COG) study D9803 protocol was applied for her [3].

She developed recurrence in her brain a few months later (Fig. 1c, d) and died at the age of 7 months.

\section{Discussion}

Massive proptosis at birth is rare. The differential diagnosis includes orbital teratoma, lymphangioma, hemangioma, meningocele, epithelial cyst, and RMS [4].

Some congenital syndromes are associated with RMS such as Li-Fraumeni syndrome (P53 gene mutation on chromosome 17p13), neurofibromatosis type 1, Noonan syndrome, Gorlin syndrome, Costello syndrome, hereditary retinoblastoma, and Beckwith-Wiedemann syndrome [5]. Our case did not have any sign of the abovementioned syndromes, and mutation in P53 was negative.

RMS treatment consists of multidrug chemotherapy, but radiotherapy and excision are sometimes indicated depending on age [6]. According to the literature, exenteration was performed in less than one-third of advanced cases or recurrence of orbital RMS at previous radiotherapy location [2]. Complete resection of the orbital mass is often difficult due to proximity of tumor to the delicate orbital structures and high risk of complications [2]. In our patient, invasion of tumor to the normal orbital structures was so severe we were not able to return the globe back at the time of surgery and preferred to do a modified exenteration.

Regardless of initial stage or group, all patients with RMS are required to be treated with chemotherapy. The standard chemotherapy is VAC regimen including three drugs (vincristine, actinomycin $\mathrm{D}$, and cyclophosphamide) [3]. Other chemotherapy agents like irinotecan, topotecan, ifosfomide, or etoposide may be used in various combinations according to the patient's clinical status and the physician's judgment [3]. With the latest che- motherapy drugs, 5-year-survival rate has risen to $92 \%$ [2]. In older children, radiotherapy is very effective to control and prevent local recurrence, but then again has little effect on survival rate [7].

Important risk factors for prognosis in various studies were age under 12 months, alveolar or undifferentiated types, location (i.e., nonorbital), size of tumor $(>5 \mathrm{~cm})$, lymph node involvement, delay in diagnosis, and presence of metastasis at the time of diagnosis [6-8]. Considering the age of our patient, undifferentiated tumor histology, large tumor size, and extraorbital invasion of the tumor, our patient had poor prognosis for survival. She had good initial response to the treatment; however, tumor recurrence led to treatment failure.

Despite advances in RMS diagnosis and treatment in recent years, it is still life-threatening and treatment is associated with lifelong complications. If any cosmetic reconstruction is needed, especially after surgical resection, adequate waiting is recommended to avoid unnecessary interventions.

In order to manage orbital RMS, it requires a multidisciplinary approach team and collaboration between pediatric ophthalmologist and oncologist.

\section{Acknowledgements}

The authors would like to thank Dr. Masoudi and his team at neurosurgery department of Shiraz Medical School for their great care during hospital admission of the baby. Also, we wish to thank Mr. H. Argasi at the Research Consultation Center (RCC) at Shiraz University of Medical sciences for his invaluable assistance in editing the manuscript.

\section{Statement of Ethics}

Informed consent was taken from the baby's parents for picture publication.

\section{Disclosure Statement}

The authors have no conflict of interest to declare. 


\section{References}

1 Rao AA, Naheedy JH, Chen JY, Robbins SL, Ramkumar HL: A clinical update and radiologic review of pediatric orbital and ocular tumors. J Oncol 2013;2013:975908.

2 Huh W, Mahajan A: Ophthalmic oncology. In: Esmaeli B (ed): Ophthalmic Oncology. Boston, Springer, 2011, pp 61-67.

3 Arndt CAS, Stoner JA, Hawkins DS, et al: Vincristine, actinomycin, and cyclophosphamide compared with vincristine, actinomycin, and cyclophosphamide alternating with vincristine, topotecan, and cyclophosphamide for intermediate-risk rhabdomyosarcoma: Children's Oncology Group study. J Clin Oncol 2009;27:5182-5188.
4 Singh AD, Traboulsi EI, Reid J, et al: Orbital cyst: prenatal diagnosis. Ophthalmology 2009;116:2042-2042.e2.

5 Kikuchi K, Rubin BP, Keller C: Developmental origins of fusion-negative rhabdomyosarcomas. Curr Top Dev Biol 2011;96:33-56.

6 Chen B, Perry JD. Rhabdomyosarcoma. In: Singh AD (ed): Clinical Ophthalmic Oncology. Philadelphia, Saunders Elsevier, 2007, pp 581-585.
7 Oberlin O, Rey A, Anderson J, et al: Treatment of orbital rhabdomyosarcoma: survival and late effects of treatment - results of an international workshop. J Clin Oncol 2001; 19:197-204.

8 Rodary C, Gehan EA, Flamant F, et al: Prognostic factors in 951 nonmetastatic rhabdomyosarcoma in children: a report from the International Rhabdomyosarcoma Workshop. Med Pediatr Oncol 1991;19:89-95. 